ORIGINAL RESEARCH

\title{
Cost Minimization Analysis of a Store-and-Forward Teledermatology Consult System
}

Hon S. Pak, M.D., ${ }^{1}$ Santanu K. Datta, Ph.D., M.B.A., ${ }^{2}$ Crystal A. Triplett, R.N., B.S.N., C.C.R.C., ${ }^{3}$ Jennifer H. Lindquist, M.Stat., ${ }^{4}$ Steven C. Grambow, Ph.D., ${ }^{5}$ and John D. Whited, M.D., M.H.S. ${ }^{2}$

'Telemedicine and Advanced Technology Research Center, Fort Detrick, Maryland.

${ }^{2}$ Center for Health Services Research in Primary Care, Veterans Affairs Medical Center, Division of General Internal Medicine, Duke University Medical Center, Durham, North Carolina.

${ }^{3}$ Department of Dermatology, Brooke Army Medical Center, Fort Sam Houston, Texas.

${ }^{4}$ Center for Health Services Research in Primary Care, Veterans Affairs Medical Center, Durham, North Carolina.

${ }^{5}$ Center for Health Services Research in Primary Care, Veterans Affairs Medical Center, Department of Biostatistics and Bioinformatics, Duke University Medical Center, Durham, North Carolina.

\section{Abstract}

The aim of this study was to perform a cost minimization analysis of store-and-forward teledermatology compared to a conventional dermatology referral process (usual care). In a Department of Defense (DoD) setting, subjects were randomized to either a teledermatology consult or usual care. Accrued healthcare utilization recorded over a 4-month period included clinic visits, teledermatology visits, laboratories, preparations, procedures, radiological tests, and medications. Direct medical care costs were estimated by combining utilization data with Medicare reimbursement rates and wholesale drug prices. The indirect cost of productivity loss for seeking treatment was also included in the analysis using an average labor rate. Total and average costs were compared between groups. Teledermatology patients incurred \$103,043 in total direct costs (\$294 average), while usual-care patients incurred \$98,365 (\$283 aver- age). However, teledermatology patients only incurred \$16,359 (\$47 average) in lost productivity cost while usual-care patients incurred $\$ 30,768$ (\$89 average). In total, teledermatology patients incurred $\$ 119,402$ (\$340 average) and usual-care patients incurred $\$ 129,133$ (\$372 average) in costs. From the economic perspective of the DoD, store-and-forward teledermatology was a cost-saving strategy for delivering dermatology care compared to conventional consultation methods when productivity loss cost is taken into consideration.

Key words: teledermatology; store-and-forward system; cost-savings

\section{Introduction}

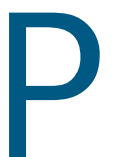

revious economic analyses of teledermatology consult systems have focused on real-time interactive modalities. ${ }^{1-7}$ Although teledermatology was found to be a cost-saving strategy compared to conventional care in some instances, more commonly, greater costs were incurred. Typically, real-time interactive technology is a more costly intervention than store-andforward consult systems, in part due to the need for more sophisticated technology requirements. Only one previous study has assessed the cost-effectiveness of a store-and-forward teledermatology consult system. ${ }^{8}$ This study found that from the economic perspective of the United States (U.S.) Department of Veterans Affairs, teledermatology was more costly than the conventional referral process, but was a cost-effective intervention.

This report is part of a randomized clinical trial designed to assess the clinical outcomes of patients undergoing store-and-forward teledermatology consultations compared to a conventional consult process in a U.S. Department of Defense (DoD) healthcare setting. We previously reported that patients undergoing a store-and-forward teledermatology consult achieve comparable clinical outcomes when compared to subjects undergoing the conventional clinic-based consult process. ${ }^{9}$ Because 


\section{COST OF STORE-AND-FORWARD TELEDERMATOLOGY CONSULT SYSTEM}

comparable clinical outcomes were found, a cost-minimization analysis comparing incurred costs between competing, yet equally effective, strategies is the analytic strategy employed for this study.

\section{Materials and Methods}

Approval for the study protocol was granted by the Institutional Review Boards of the Brooke Army Medical Center (BAMC), Fort Sam Houston, Texas, and the Veterans Affairs Medical Center, Durham, North Carolina. All subjects provided written informed consent.

Details of recruitment, study procedures, and clinical outcomes are detailed in a previous publication. ${ }^{9}$ Briefly, participating subjects who were being referred from a Primary Care Clinic to a Dermatology Clinic served by the BAMC or the Darnall Army Community Hospital, Fort Hood, Texas and affiliated clinics were randomized to either a store-and-forward teledermatology consult or a conventional consult process (usual care). The store-and-forward teledermatology consult consisted of a set of digital images and a standardized history obtained per protocol. This information was forwarded to a dermatologist who reviewed the teledermatology consult and sent recommendations back to the referring clinician that could include a recommendation that the patient be evaluated in a Dermatology Clinic. Those patients who were randomized to usual care were scheduled for a Dermatology Clinic visit, the normal course of care for the conventional referral process. Clinical outcomes were assessed by obtaining a baseline set of images and a second set of images 4 months later in both study groups. A dermatologist, blinded to study group assignment, made the clinical course assessment by serial image review by rating the clinical course as worse, no change, or improved. Likewise, costs were recorded over the 4-month period of patient participation. Because comparable clinical outcomes were found, a cost minimization analysis that reports comparative costs of two equally effective competing strategies was performed. The economic perspective we took was that of the DoD; thus, those costs that would be borne by the DoD were included in the analysis.

Costs data relied, in part, on patient-reported data during the course of their participation. Patients were queried at their 4-month visit (imaging visit for the clinical course assessment) about clinic visits related to their referred skin condition as well as any procedures they may have undergone. We did not have access to and did not collect encounter form data (i.e., the form clinicians use when assessing the charges related to a particular clinic visit). Thus, we made simplifying assumptions to estimate costs (e.g., assigned Current Procedural and Terminology [CPT] codes) as indicated in Table 1. The occurrence of a clinic visit, however, was confirmed by a medical record review that provided the record of a clinic visit occurrence but not the associated charges generated from that visit. Medications, recommendations for laboratory tests, biopsies, and so on were recorded based on the dermatologists' recommendations after the consultations (teledermatology or usual care) were completed.

Direct costs were calculated using the costs of clinic visits, teledermatology encounters, radiology procedures, laboratories, preparations (e.g., KOH preparation), procedures (e.g., biopsies), and medications (Table 1). ${ }^{10-12}$ The cost of generating and reviewing a teledermatology consult was captured as the reimbursement rate for a 99242 Evaluation and Management Consultation code using 2006 data. ${ }^{11}$ Clinic visit costs were also based on a reimbursement rate using the 99242 Evaluation and Management Consultation code. We used Medicare payment rates based on total nonfacility relative value units (RVUs), which includes the physician's work component, the practice expense component, and the professional liability insurance component. Medicare has two different levels of practice expense RVUs, facility and nonfacility. Facility includes such settings as hospitals (inpatient, outpatient, and emergency department), ambulatory surgical centers, and skilled nursing facilities; nonfacility includes all other settings. Because dermatology procedures are likely to be performed in nonfacility settings, we chose to use nonfacility RVUs. Visits and consultations included the cost of all visits made during the 4-month follow-up period that were related to the referred skin complaint. Other cost data, as appropriate, were based on CPT coding or the Center for Medicare and Medicaid Services 2006 Clinical Diagnostic Laboratory Fee Schedule. ${ }^{11,12}$ "Other laboratories and procedures" were collected and reported separately from those laboratories and procedures that were expected to be frequently incurred a priori and were included to provide a more accurate representation of total resource utilization. Medication costs were based on average wholesale prices. ${ }^{10}$ Medication names, dosing, frequency, and dispensed amount were included in the database. We did not attempt to collect data on refills; therefore, medication costs included the cost of medications dispensed at the initial fill. Medications available over the counter were not included in the analysis. If dosing and other items were not specified, by convention, we used the lowest specified dosing regimen or amount.

Indirect costs were calculated based on lost productivity. Accounting for productivity loss is advocated by The Panel on Cost-Effectiveness in Health and Medicine. ${ }^{13}$ Since lost productivity is a cost that would be incurred by the $\mathrm{DoD}$, we included these data a priori in the analysis. We considered that each in-person clinic visit (dermatology or other) would incur, on average, 4 hours of lost productivity that would be required to attend the visit and travel to and from the visit. We excluded the baseline visit that generated the dermatology consult. All patients incurred a baseline visit and, therefore, no incremental cost difference between study groups is expected. Using 2006 
PAK ET AL.

Table 1. Resource Utilization and Cost Data Used in the Analysis

RESOURCE UTILIZATION CPT OR OTHER CODES ${ }^{a}$

$\operatorname{cosT}$

COMMENTS

Consultations/visits

\begin{tabular}{|c|c|c|c|}
\hline Dermatology consultation or other specialty consultation & 99242 & $\$ 87.91$ & \\
\hline Teledermatology consultation & 99242 & $\$ 87.91$ & \\
\hline Primary care visit & 99213 & $\$ 50.29$ & \\
\hline \multicolumn{4}{|l|}{ Procedures } \\
\hline Punch biopsy & 11100 & $\$ 75.61$ & First biopsy used for all procedures \\
\hline Shave biopsy & 11100 & $\$ 75.61$ & First biopsy used for all procedures \\
\hline Excision biopsy & 11400 & $\$ 105.28$ & Benign or not known used for all procedures \\
\hline Mohs surgery & 17304 & $\$ 584.26$ & \\
\hline Cryotherapy & 17340 & $\$ 42.69$ & \\
\hline Laser therapy & 17106 & $\$ 345.13$ & \\
\hline Electrodessication and curettage & 1700 & $\$ 57.88$ & \\
\hline UV therapy & 96913 & $\$ 64.40$ & \\
\hline
\end{tabular}

\begin{tabular}{l|c|c|c}
\hline \multicolumn{3}{l}{} \\
Labs/preparations \\
\hline KOH prep & 87220 & $\$ 19.54$ & \\
\hline Gram stain & 87205 & $\$ 19.54$ & \\
\hline Tzank prep & 88104 & $\$ 29.67$ & \\
\hline CBC & HCPC 85025 & $\$ 10.86$ & \\
\hline Chem 7 & HCPC 80053 & $\$ 9.80$ & \\
\hline Liver panel & HCPC 80076 & $\$ 11.42$ & \\
\hline TSH (thyroid) & HCPC 84443 & $\$ 23.47$ & ECG (rhythm), w/report \\
\hline ECG & 93042 & $\$ 7.96$ & \\
\hline
\end{tabular}

Other labs and procedures

ANA, ENA, RF, RPR, ESR, lipid panels, fungal cultures, beta hCG, hepatitis serologies, immunofluorescence, PT/PTT, iron, ferritin, viral and bacterial cultures, wound cultures, intralesional injections, patch testing, glycolic peel, extractions, sclerotherapy, suture removal, hypercator, ACE levels, urinalysis, FSH, LH, testosterone, and DHEA-SO4

\begin{tabular}{l|l|l|l}
\hline Radiology & \multicolumn{2}{l}{} \\
\hline MRI right foot & 73718 & $\$ 487.30$ & MRI, lower extrem. w/o dye \\
\hline CT orbit & 70480 & $\$ 243.83$ & CT orbit/ear/fossa w/o dye \\
\hline AP/lateral spine & 72010 & $\$ 61.50$ & X-ray exam of spine \\
\hline Chest x-ray-PA/lateral & 71020 & $\$ 34.37$ & \\
\hline Upper Gl series & 74240 & $\$ 90.09$ & \\
\hline MRI cervical spine & 72141 & $\$ 507.20$ & MRI neck spine w/o dye \\
\hline
\end{tabular}

\section{Medications}

Based on 2005 Red Book data ${ }^{10}$

aSources were Medicare RBRVS: The Physicians' Guide, 2006 for Evaluation and Management Codes and Current Procedural Terminology ${ }^{\circledR}$ codes or CMS 2006 Clinical Diagnostic Laboratory Fee Schedule, National. Available at: http://www.cms.hhs.gov/ClinicalLabFeeSched/02_clinlab.asp.

UV, ultraviolet; CBC, complete blood count; TSH, thyroid stimulating hormone; ANA, antinuclear antibody; ENA, extractable nuclear antigen; RF, rheumatoid factor; RPR, rapid plasma reagin; ESR, erythrocyte sedimentation rate; hCG, human chorionic gonadotropin; PT, prothrombin time; PT, partial thromboplastin time; ACE, angiotensin-converting enzyme; FSH, follicle-stimulating hormone; $\mathrm{LH}$, luteinizing hormone; DHEA, dehydroepiandrosterone; $\mathrm{MRI}$, magnetic resonance imaging; $\mathrm{CT}$, computed tomography;

$A P$, anterior-posterior; PA, posterior-anterior; GI, gastrointestinal. 
data from the Bureau of Labor Statistics for the San Antonio, Texas area, we assigned 4 hours of lost wages at the hourly rate of $\$ 15.73$ as the cost of lost productivity. ${ }^{14}$

Costs are reported as total group costs and average group costs. Costs were calculated using observed outcomes and events. Only those clinic visits, procedures, and laboratories that occurred, as opposed to those recommended, and only those medications that were filled were included in the cost analysis. Since we were using the economic perspective of the DoD, cost reporting also used an intentto-treat type analysis. All patients who randomized were included in the cost analysis regardless of whether they completed follow-up over the 4-month period. Costs are reported in 2006 dollars.

\section{Results}

Three hundred fifty-one $(n=351)$ subjects randomized to teledermatology (Group 1) and $347(n=347)$ subjects randomized to usual care (Group 2). As might be expected, more dermatology clinic visits were encountered in the usual-care arm and more primary care visits occurred in Group 1. For Group 1, 208 dermatology clinic visits, 42 primary care visits, and 10 other specialty clinic visits occurred. The 208 dermatology clinic visits in teledermatology occurred among 121 patients (34.5\% of those randomized to teledermatology). Among the 121 teledermatology patients 61\% percent (74/121) were seen for a single dermatology clinic visit, 22\% (26/121) had two visits, $8 \%$ $(10 / 121)$ had three visits, 4\% (5/121) had four visits, 3\% (4/121) had

\begin{tabular}{l|c|c}
\multicolumn{3}{c}{ Table 2. Cost Minimization Analysis Results } \\
COST ELEM ENT & $\begin{array}{c}\text { TELEERMATOLOGY } \\
(N=351)\end{array}$ & $\begin{array}{c}\text { USUAL CARE } \\
(N=347)\end{array}$ \\
\hline $\begin{array}{l}\text { Clinic-based visits } \\
\text { Dermatology }\end{array}$ & $\$ 21,276(\$ 61)$ & $\$ 42,837(\$ 123)$ \\
Primary care & $\$ 18,285$ & $\$ 41,669$ \\
Other & $\$ 2,112$ & $\$ 879$ \\
\hline Teledermatology consultations & $\$ 30,856(\$ 88)$ & $\$ 967$ \\
\hline Procedures/labs/preparations & $\$ 15,020(\$ 43)$ & - \\
\hline Other labs/procedures & $\$ 4,538(\$ 13)$ & $\$ 21,103(\$ 61)$ \\
\hline Radiology & $\$ 521(\$ 1)$ & $\$ 937(\$ 3)$ \\
\hline Medications & $\$ 30,832(\$ 88)$ & $\$ 27,375(\$ 79)$ \\
\hline Total direct cost (average cost) & $\$ 103,043(\$ 294)$ & $\$ 98,365(\$ 283)$ \\
\hline Lost productivity & $\$ 16,359(\$ 47)$ & $\$ 30,768(\$ 89)$ \\
\hline Total cost (average cost) & $\$ 119,402(\$ 340)$ & $\$ 129,133(\$ 372)$ \\
\hline
\end{tabular}

(\$), average cost per patient. five visits, and 2\% (2/121) had six visits. Of note, due to the limited capability of the referring providers in this setting, if any procedures (e.g., cryotherapy, biopsies, etc.) were recommended by the teledermatologist most, if not all, of these patients were sent to the dermatology clinic. For Group 2, 474 dermatology clinic visits, 4 primary care visits, and 11 other specialty clinic visits occurred.

Total and average costs for each group appear in Table 2. Total costs for clinic-based visits were higher in usual care compared to teledermatology: $\$ 42,837$ versus $\$ 21,276$, respectively. These lower clinic-based costs for teledermatology are offset by the cost incurred to perform the teledermatology consultations $(\$ 30,856)$. This cost element, of course, does not apply to usual care. Overall, the sum cost of procedures, laboratories, preparations, radiology procedures, and medications were fairly similar $(\$ 50,911$ for teledermatology and $\$ 55,528$ for usual care). Greater costs were observed for laboratories, procedures, and radiological tests in usual care, whereas teledermatology generated greater medication costs.

Total and average direct costs were slightly higher for teledermatology compared to usual care. The total direct cost of teledermatology was $\$ 103,043$ and the average cost was $\$ 294$. For usual care, total direct cost was $\$ 98,365$ and the average cost was $\$ 283$. Thus, the average direct cost of teledermatology was $\$ 11$ higher than that of usual care.

Teledermatology gained an economic advantage when loss of productivity costs was considered. Lost productivity costs were $\$ 16,359$ for teledermatology compared to $\$ 30,768$ for usual care. This resulted in teledermatology becoming the cost-saving strategy with a total cost of $\$ 119,402$ ( $\$ 340$ average cost) compared to total cost for usual care of $\$ 129,133$ (\$372 average cost). The average total cost of teledermatology was $\$ 32$ less than that found for usual care.

\section{Discussion}

Teledermatology was a cost-saving strategy compared to the conventional referral process. Teledermatology resulted in fewer clinicbased dermatology visits, but that cost advantage was lost by the cost incurred to generate and review the teledermatology consult. Greater expenditures were noted for laboratories, procedures, and radiology studies in usual care, yet greater medication expenditures were found in Group 1. The direct costs were fairly similar between randomization groups, with slightly lower costs found for Group 2. However, loss of productivity costs for usual care were approximately double that found in teledermatology, resulting in teledermatology becoming the cost-saving strategy. These results are consistent with emerging evidence that when telemedicine is a cost-saving strategy, much of the cost avoidance is accrued from averted travel, less time lost from employment, averted patient transfer, or similar cost elements. ${ }^{4-6,8}$ 
We chose to conservatively estimate the cost of performing a teledermatology consult using the same Evaluation and Management Consultation code that would be used for a clinic-based visit. It is likely that average reimbursement rates for clinic-based visits would exceed our estimate. Furthermore, we likely are underestimating the cost savings of teledermatology since most patients requiring procedures were referred to a dermatologist rather than being managed by the referring primary care provider. In other settings, many minor procedures (e.g., cryotherapy and shave biopsies) are performed by a primary care provider. It could also be argued that the cost to perform teledermatology consults in a setting such as the DoD healthcare system could be considered a "sunk cost." This implies that much, if not all, of the cost of performing teledermatology consultations is already borne by the healthcare system and adds little, if any, additional costs. We did not perform an analysis using this assumption, nor did we consider the assumptions that store-and-forward teledermatology consultations may ultimately be reimbursed at lower rates than clinic-based visits or that average clinic-based reimbursement may be higher than the value we used. These assumptions, however, would only serve to increase the magnitude of the observed total cost savings found in this study.

It is postulated that teledermatology may "miss" skin conditions that otherwise may be found in a clinic-based examination. Our study was not designed to quantify this occurrence, and our results add little clarity to this question. Greater procedural costs were found in usual care, whereas teledermatology incurred greater medication costs. If more disease is found in usual care, then one might expect both greater procedural and medication costs. In any event, this is a potentially important area for future investigation.

Our study has limitations. First, although our study population was diverse and likely represented a wide range of dermatologic conditions, our results may not be generalizable to other populations. To the extent that other populations would be expected to incur greater or fewer clinic-based visits, particularly as a result of teledermatology consultations, the outcomes may differ. For example, if teledermatology is used in a population with a high prevalence of skin cancer, a large proportion of teledermatology consults could result in clinic-based visits for biopsy procedures, thus averting fewer clinic visits than our observations. Alternatively, a population that can largely be managed by the referring clinician on the basis of recommendations of the teledermatologist may realize greater cost savings. Second, our cost data collection relied, in part, on patient self-report. Although patient-reported data were verified by medical record review, it is possible that some cost data were not recorded. This could have influenced some of the differ- ences we found in the costs of laboratories, procedures, and medications between the two study groups. Additionally, interobserver differences and different practice styles among the dermatologists performing the consultations likely influenced these differences between study groups. Future studies specifically designed to assess treatment choices between teledermatologists and in-person dermatologists may lend better clarity to this question. Third, we made necessary simplifying assumptions to estimate some cost elements such as the cost of a store-and-forward teledermatology encounter, CPT coding of procedures, laboratories, and radiology procedures. Fourth, the intent-to-treat approach allows us to report group average costs but not the standard deviations or confidence intervals around these average costs.

Our previous study found that store-and-forward teledermatology results in comparable clinical outcomes as measured by 4-month clinical course assessments. ${ }^{9}$ This report indicates that store-and-forward teledermatology delivers these comparable clinical outcomes at moderate cost savings, with an average cost per subject of $\$ 340$ for teledermatology versus $\$ 372$ per subject in usual care: a difference of $\$ 32$.

\section{Acknowledgments}

This study was funded by the U.S. Army's Telemedicine and Advanced Technology Research Center, Fort Detrick, Maryland.

\section{Disclaimer}

The views expressed in this article are those of the authors and do not necessarily represent the views of the Department of Defense or the Department of Veterans Affairs.

\section{Disclosure Statement}

HSP is Chairman and Co-founder of TeledermSolutions, Inc., a Web-based teledermatology consultation service, is Co-editor of Teledermatology: A user's guide, published by Cambridge University Press, and is slated to receive royalties based on sales. SKD: No competing financial interest exists. CAT: No competing financial interest exists. JHL: No competing financial interest exists. SCG: No competing financial interest exists. JDW is Co-editor of Teledermatology: A user's guide, published by Cambridge University Press, and is slated to receive royalties based on sales.

\section{REFERENCES}

1. Loane MA, Bloomer SE, Corbett R, Eedy DJ, Evans C, Hicks N, et al. A randomized controlled trial assessing the health economics of real-time teledermatology compared with conventional care: An urban versus rural perspective. J Telemed Telecare 2001;7:108-118. 


\section{COST OF STORE-AND-FORWARD TELEDERMATOLOGY CONSULT SYSTEM}

2. Wootton $\mathrm{R}$, Bloomer SE, Corbett R, Eedy DJ, Hicks N, Lotery HE, et al. Multicentre randomized control trial comparing real time teledermatology with conventional outpatient dermatological care: A societal cost-benefit analysis. BMJ 2000;320:1252-1256.

3. Lamminen $H_{\text {, Tuomi }} M-L$, Lamminen J, Uusitalo $H$. A feasibility study of realtime teledermatology in Finland. J Telemed Telecare 2000;6:102-107.

4. Loane MA, Oakley A, Rademaker M, Bradford N, Fleischl P, Kerr P, et al. A cost-minimization analysis of the societal costs of real-time teledermatology compared with conventional care: Results from a randomized controlled trial in New Zealand. J Telemed Telecare 2001;7:233-238.

5. Bergmo TS. A cost-minimization analysis of a realtime teledermatology service in northern Norway. J Telemed Telecare 2000;6:273-277.

6. Chan HHL, Woo J, Chan WM, Hjelm M. Teledermatology in Hong-Kong: A costeffective method to provide service to the elderly patients living in institutions. Int J Dermato/ 2000;39:774-778.

7. Persaud DD, Jreige S, Skedgel C, Finley J, Sargeant J, Hanlon N. An incremental cost analysis of telehealth in Novia Scotia from a societal perspective. J Telemed Telecare 1995;11:77-84.

8. Whited JD, Datta S, Hall RP, Foy ME, Marbrey LE, Grambow SC, et al. An economic analysis of a store-and-forward teledermatology consult system. Telemed J 2003;9:351-360.

9. Pak H, Triplett CA, Lindquist JH, Grambow SC, Whited JD. Store and forward teledermatology results in comparable clinical outcomes compared to conventional clinic-based care. J Telemed Telecare 2007;13:26-30.
10. 2005 Redbook. Montvale, NJ: Thomson PDR, 2005.

11. Medicare RBRVS: The physicians' guide 2006. Chicago: American Medical Association, 2006:464.

12. CMS 2006 Clinical Diagnostic Laboratory Fee Schedule, National. Available at: http:// www.cms.hhs.gov/ClinicalLabFeeSched/02_clinlab.asp (Last accessed June 26, 2006).

13. Gold MR, Siegel JE, Russell LB, Weinstein MC. Cost effectiveness in health and Medicine. Oxford University Press, New York, 1996.

14. United States Department of Labor. San Antonio TX, National Compensation Survey November 2005. United States Bureau of Labor Statistics, March 2006. Available at: http://stats.bls.gov/ncs/ocs/sp/ncbl0785.pdf (Last accessed September 26, 2006).

Address reprint requests to: Santanu K. Datta, Ph.D., M.B.A.

VA Medical Center (152) 508 Fulton Street Durham, NC 27705

E-mail:datta001@mc.duke.edu

Received: June 10, 2008 Accepted: August 3, 2008 